Supporting Information for:

\title{
Observation and Analysis of Incoherent Second-Harmonic Generation in Gold Nanoclusters with Six Atoms
}

Renato Barbosa-Silva ${ }^{1,}$, Manoel L. Silva-Neto ${ }^{2}$, Dipankar Bain ${ }^{3}$, Lucas Modesto-Costa ${ }^{4,5}$, Tarciso Andrade-Filho ${ }^{6}$, Vinícius Manzoni ${ }^{7}$, Amitava Patra ${ }^{3}$, and Cid B. de Araújo ${ }^{1,2}$

${ }^{1}$ Departamento de Física, Universidade Federal de Pernambuco, 50670-901, Recife, Brazil.

2 Programa de Pós-Graduação em Ciência de Materiais, Universidade Federal de Pernambuco, 50670-901, Recife, PE, Brazil.

${ }^{3}$ School of Materials Sciences, Indian Association for the Cultivation of Science, 700032, Kolkata, India.

${ }^{4}$ Departamento de Física, Universidade Federal de Juiz de Fora, 36036-330 Juiz de Fora, MG, Brazil.

${ }^{5}$ Departamento de Química, Instituto Militar de Engenharia, 22291-270, Rio de Janeiro, RJ, Brazil.

${ }^{6}$ Faculdade de Física, Universidade Federal do Sul e Sudeste do Pará, 68505-080 Marabá, PA, Brazil.

${ }^{7}$ Instituto de Física, Universidade Federal de Alagoas, 57072-900 Maceió, AL, Brazil.

*Corresponding author. E-mail: renatobsilva@gmail.com 
INSTRUMENTATION: The transmission electron microscopy (TEM) was recorded using JEOL-JEM-2100F transmission electron microscopy system with accelerating voltage of $200 \mathrm{kV}$. The photoluminescence (PL) spectrum of Au NCs was measured using Fluoro Max-P (HORIBA Jobin Yvon) luminescence spectrophotometer. Mass of Au NCs was analyzed using matrixassisted laser desorption ionization-time-of-flight (MALDI-TOF) mass spectrometry (Bruker Daltonics Autoflex II TOF/TOF). A pulsed laser of $337 \mathrm{~nm}$ was used and a saturated matrix solution $\alpha$-Cyano-4-hydroxycinnamic acid (CHCA) was selected for the MALDI-TOF experiment. For the time correlated single photon counting (TCSPC) analysis, the sample was excited at $371 \mathrm{~nm}$ using picosecond Nano-LED 370L instrument. The fluorescence decay was collected on a Hamamatsu MCP photomultiplier. The experimental time-resolved luminescence decays were analyzed using equation (1):[1]

$$
P(t)=b+\sum_{i}^{n} \alpha_{i} \exp \left(-t / \tau_{i}\right) .
$$

Here, $\mathrm{n}$ is the number of emissive species, $\mathrm{b}$ is the baseline correction ("DC" offset), and $\alpha_{\mathrm{i}}$ and $\tau_{\mathrm{i}}$ are, respectively, the pre-exponential factor and the excited-state fluorescence decay time associated with the $i^{\text {th }}$ component. The average decay time $<\tau>$ is estimated from equation (2).

$$
<\tau>=\sum_{i=1}^{n} \beta_{i} \tau_{i},
$$

where $\beta_{i}=\alpha_{i} / \sum \alpha_{i}$ and $\beta_{i}$ is the contribution of the decay component.

An ultrafast time-resolved spectroscopic measurement was carried out using a femtosecond luminescence upconversion spectrophotometer with a Halcyone ultrafast setup. The Au NCs were excited at $400 \mathrm{~nm}$ wavelength, pumped using a $800 \mathrm{~nm}$ fs (140 fs pulse width, $80 \mathrm{MHz}$ repetition rate) laser pulse $(4.4 \mathrm{~W})$ from a Ti:sapphire oscillator (Chameleon, Coherent) coupled to a second harmonic generator (by BBO type I crystal). Using a nonlinear crystal (BBO type II), the emission wavelength $(512 \mathrm{~nm})$ and the gate pulse of the fundamental beam $(800 \mathrm{~nm})$ were upconverted. The full-width-at-half-maximum (FWHM) of the instrument response function was about $288 \mathrm{fs}$. Surface Xplorer 4 fitting software was employed to fit the femtosecond time-resolved decay data. 
MALDI-TOF SPECTRUM OF THE NANOCLUSTERS

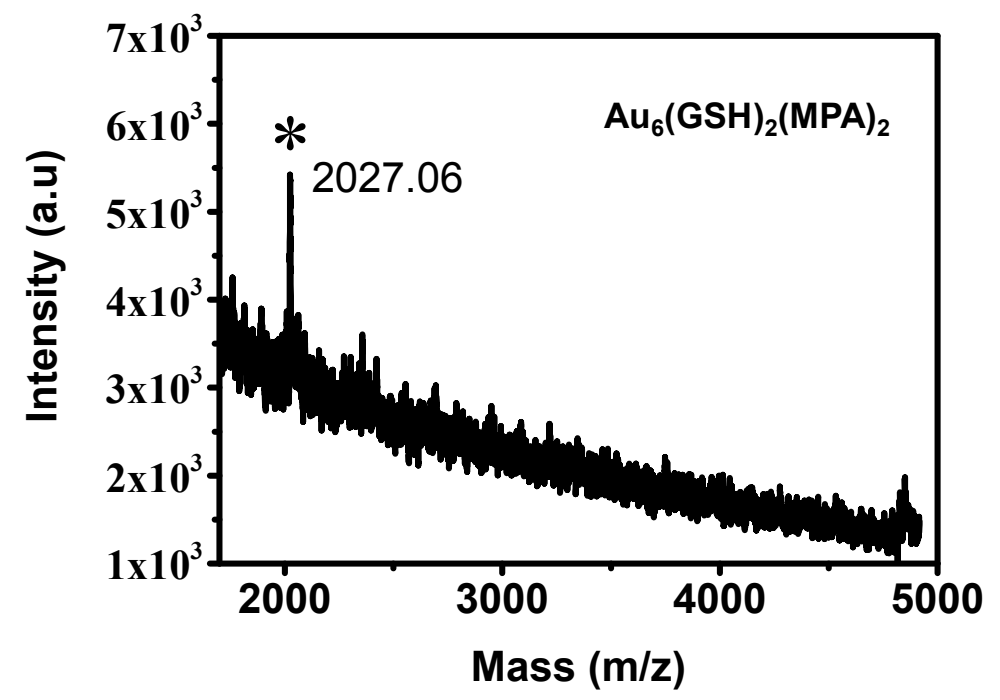

Figure S1. MALDI-TOF spectrum of Au NCs. a.u. stands for arbitrary units.

\section{WAVELENGTH DEPENDENCE OF THE NCS PHOTOLUMINESCENCE}

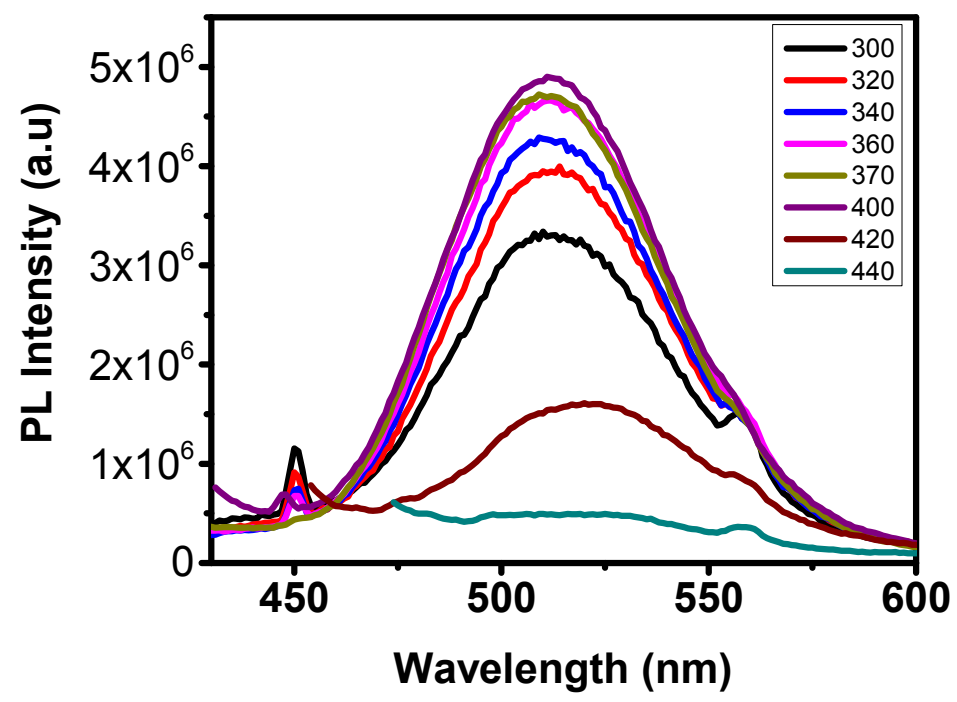

Figure S2. Emission spectra of Au NCs for different excitation wavelengths. a.u. stands for arbitrary units. 
Table S1. Theoretical values of calculated components of the hyperpolarizability tensor obtained with DFT wB97XD methodology in gas phase. Basis set: Au atoms LANL2DZ; S C N O H atoms $6-311+G(d, p)$.

\begin{tabular}{|c|c|c|c|}
\hline \multicolumn{4}{|c|}{$\beta(0 ; 0,0):$} \\
\hline & (au) & $(10 * *-30$ esu) & $(10 * *-50 \mathrm{SI})$ \\
\hline $\mathrm{xxx}$ & $-0.211258 D+04$ & $-0.182511 D+02$ & $-0.677371 D+01$ \\
\hline$x x y$ & $-0.868567 D+03$ & $-0.750374 D+01$ & $-0.278494 D+01$ \\
\hline yyy & $0.149779 D+04$ & $0.129398 \mathrm{D}+02$ & $0.480246 \mathrm{D}+01$ \\
\hline$x x z$ & $0.593634 \mathrm{D}+02$ & $0.512853 D+00$ & $0.190340 D+00$ \\
\hline$y x z$ & $-0.409800 D+03$ & $-0.354035 D+01$ & $-0.131397 D+01$ \\
\hline yyz & $0.476270 D+03$ & 0.411461D+01 & $0.152710 D+01$ \\
\hline$z X z$ & $-0.162128 D+04$ & $-0.140066 \mathrm{D}+02$ & $-0.519842 \mathrm{D}+01$ \\
\hline zyz & $0.566213 D+03$ & 0.489164D+01 & $0.181548 D+01$ \\
\hline $\mathrm{zzz}$ & $0.918255 \mathrm{D}+03$ & $0.793300 D+01$ & $0.294426 \mathrm{D}+01$ \\
\hline \multicolumn{4}{|c|}{$\beta(-2 \omega ; \omega, \omega) ; \omega=2 \pi c / \lambda ; \lambda=1064.0 \mathrm{~nm} ; c=3 \times 10^{8}: \mathrm{m} / \mathrm{s}$} \\
\hline & (au) & $(10 * *-30$ esu $)$ & $(10 * *-50 \mathrm{SI})$ \\
\hline$x x x$ & $-0.176646 D+04$ & $-0.152609 D+02$ & $-0.566392 D+01$ \\
\hline$y x x$ & $0.317550 \mathrm{D}+05$ & $0.274339 D+03$ & $0.101818 D+03$ \\
\hline zxx & $0.219913 D+05$ & $0.189988 \mathrm{D}+03$ & $0.705122 \mathrm{D}+02$ \\
\hline xyx & $-0.227459 D+05$ & $-0.196507 D+03$ & $-0.729316 D+02$ \\
\hline yyx & $0.412140 \mathrm{D}+05$ & $0.356057 \mathrm{D}+03$ & $0.132147 \mathrm{D}+03$ \\
\hline zyx & $0.106347 D+05$ & $0.918758 \mathrm{D}+02$ & $0.340988 D+02$ \\
\hline xyy & $-0.101043 D+06$ & $-0.872929 D+03$ & $-0.323979 D+03$ \\
\hline yyy & $0.106975 D+06$ & $0.924182 \mathrm{D}+03$ & $0.343001 D+03$ \\
\hline zyy & $-0.621167 D+04$ & $-0.536640 D+02$ & $-0.199169 D+02$ \\
\hline$x z x$ & $0.122992 \mathrm{D}+05$ & $0.106255 D+03$ & $0.394356 \mathrm{D}+02$ \\
\hline$y z x$ & $-0.270951 D+04$ & $-0.234081 D+02$ & $-0.868768 D+01$ \\
\hline$z z x$ & $0.820411 D+04$ & $0.708771 \mathrm{D}+02$ & $0.263053 \mathrm{D}+02$ \\
\hline$x z y$ & $-0.853115 D+02$ & $-0.737025 D+00$ & $-0.273539 D+00$ \\
\hline yzy & $0.406723 D+05$ & $0.351377 D+03$ & $0.130410 D+03$ \\
\hline zzy & $0.274445 \mathrm{D}+05$ & $0.237099 D+03$ & $0.879971 \mathrm{D}+02$ \\
\hline$x z z$ & $-0.900219 D+05$ & $-0.777719 D+03$ & $-0.288643 D+03$ \\
\hline yzz & $0.122098 D+06$ & $0.105483 D+04$ & $0.391490 D+03$ \\
\hline $\mathrm{ZzZ}$ & $0.116662 D+05$ & $0.100787 D+03$ & $0.374062 \mathrm{D}+02$ \\
\hline
\end{tabular}


Table S2. Theoretical values of calculated components of the hyperpolarizability tensor obtained with DFT wB97XD methodology in water using IEF-PCM. Basis set: Au atoms LANL2DZ; S C $\mathrm{N} \mathrm{O} \mathrm{H}$ atoms $6-311+\mathrm{G}(\mathrm{d}, \mathrm{p})$.

\begin{tabular}{|c|c|c|c|}
\hline \multicolumn{4}{|c|}{$\beta(0 ; 0,0):$} \\
\hline & (au) & $(10 * *-30$ esu $)$ & $(10 * *-50 \mathrm{SI})$ \\
\hline$x \mathrm{Xx}$ & $-0.529086 \mathrm{D}+04$ & $-0.457089 D+02$ & $-0.169644 D+02$ \\
\hline$x x y$ & $-0.118522 D+03$ & $-0.102394 D+01$ & $-0.380025 D+00$ \\
\hline yxy & $-0.568511 D+04$ & $-0.491149 D+02$ & $-0.182285 \mathrm{D}+02$ \\
\hline yyy & $0.190160 \mathrm{D}+04$ & $0.164284 \mathrm{D}+02$ & $0.609722 \mathrm{D}+01$ \\
\hline$x x z$ & $0.117472 D+02$ & $0.101487 D+00$ & $0.376658 \mathrm{D}-01$ \\
\hline$y x z$ & $0.139259 D+04$ & $0.120309 D+02$ & $0.446515 \mathrm{D}+01$ \\
\hline yyz & $0.392748 D+04$ & $0.339304 \mathrm{D}+02$ & $0.125929 D+02$ \\
\hline$z x z$ & $-0.784372 D+03$ & $-0.677636 \mathrm{D}+01$ & $-0.251498 D+01$ \\
\hline zyz & $-0.957859 D+02$ & $-0.827515 D+00$ & $-0.307124 D+00$ \\
\hline $\mathrm{zzz}$ & $0.797991 \mathrm{D}+04$ & $0.689402 D+02$ & $0.255865 \mathrm{D}+02$ \\
\hline \multicolumn{4}{|c|}{$\beta(-2 \omega ; \omega, \omega) ; \omega=2 \pi c / \lambda ; \lambda=1064.0 \mathrm{~nm} ; c=3 \times 10^{8}: \mathrm{m} / \mathrm{s}$} \\
\hline & (au) & $\left(10^{* *}-30\right.$ esu $)$ & $(10 * *-50 \mathrm{SI})$ \\
\hline$x x x$ & $-0.454842 D+04$ & $-0.392948 D+02$ & $-0.145839 D+02$ \\
\hline yxx & $0.870010 \mathrm{D}+03$ & $0.751621 \mathrm{D}+01$ & $0.278957 \mathrm{D}+01$ \\
\hline zxx & $-0.250721 D+04$ & $-0.216604 D+02$ & $-0.803903 D+01$ \\
\hline xyx & $0.482378 \mathrm{D}+03$ & $0.416737 D+01$ & $0.154668 \mathrm{D}+01$ \\
\hline yyx & $-0.539705 D+04$ & $-0.466263 D+02$ & $-0.173049 D+02$ \\
\hline zyx & $0.212816 \mathrm{D}+04$ & $0.183857 D+02$ & $0.682366 \mathrm{D}+01$ \\
\hline xyy & $-0.428806 \mathrm{D}+04$ & $-0.370455 D+02$ & $-0.137491 D+02$ \\
\hline yyy & $0.967295 \mathrm{D}+04$ & $0.835667 D+02$ & $0.310150 \mathrm{D}+02$ \\
\hline zyy & $0.127877 D+04$ & $0.110476 \mathrm{D}+02$ & $0.410021 \mathrm{D}+01$ \\
\hline$x z x$ & $-0.694766 \mathrm{D}+03$ & $-0.600224 D+01$ & $-0.222767 D+01$ \\
\hline$y z x$ & $0.252822 \mathrm{D}+04$ & $0.218418 \mathrm{D}+02$ & $0.810638 \mathrm{D}+01$ \\
\hline$z z x$ & $-0.149763 D+04$ & $-0.129383 D+02$ & $-0.480193 D+01$ \\
\hline$x z y$ & $0.270024 \mathrm{D}+04$ & $0.233280 \mathrm{D}+02$ & $0.865795 \mathrm{D}+01$ \\
\hline yzy & $0.902866 \mathrm{D}+04$ & $0.780006 \mathrm{D}+02$ & $0.289491 \mathrm{D}+02$ \\
\hline zzy & $0.254338 \mathrm{D}+04$ & $0.219729 D+02$ & $0.815501 \mathrm{D}+01$ \\
\hline $\mathrm{XzZ}$ & $0.508004 \mathrm{D}+03$ & $0.438876 \mathrm{D}+01$ & $0.162884 \mathrm{D}+01$ \\
\hline yzz & $0.203739 D+04$ & $0.176015 \mathrm{D}+02$ & $0.653262 D+01$ \\
\hline $\mathrm{zzz}$ & $0.587438 \mathrm{D}+04$ & $0.507501 \mathrm{D}+02$ & $0.188354 \mathrm{D}+02$ \\
\hline
\end{tabular}

\section{Reference:}

[1] Lakowicz, J. R. Principles of Fluorescence Spectroscopy, 3rd ed.; Springer, 2006. 